59.

\section{The Epidemiology of Bridge Jumpers}

Patti Purpura, ${ }^{1}$ Jane H. Brice, $M D, 2 *$ Theodore Delbridge, $M D$,

$M P H,{ }^{3}$ Abdulrezzak Shakir, $M D,{ }^{4}$ Laurie Byme, $M D,{ }^{5}$

Robert McCaughan, EMT-P, ${ }^{6}$ Cyril Wecht, MD, $J D,{ }^{4}$

Joanne Kuntz, $M D^{7}$

1. University of Pittsburgh, School of Medicine, Pittsburgh

Pennsylvania USA

2. University of Pittsburgh Affiliated Residency in Emergency

Medicine, Pittsburgh Pennsylvania USA

3. Department of Emergency Medicine, University of Pittsburgh,

Pittsburgh, Pennsylvania USA

4. Allegheny County Coroner's Office

5. Department of Emergency Medicine, Allegheny General

Hospital, Pittsburgh, Pennsylvania USA

6. Bureau of EMS, City of Pittsburgh, Pennsylvania USA

7. Mercy Hospital of Pittsburgh, Pittsburgh, Pennsylvania USA

Purpose: Treatment and prevention strategies regarding people who jump from medium height bridges over water could be optimized by an improved understanding of patient demographics and injury spectra. Currently, little is known about this EMS patient population. We sought to describe the demographics and injuries sustained by those who jumped or fell from medium height bridges.

Methods: We searched the River Rescue and EMS reports of a medium size city for the ten year period 1986-1995 to identify cases involving a person who jumped/fell from a bridge into water. Additional cases were identified by searching coroner and trauma center registries. For each case, we reviewed applicable EMS, hospital, and coroner records to determine patient demographics, treatment provided, and injuries sustained.

Results: We identified 76 cases of individuals who jumped/fell from bridges into water. Eighteen of the region's 24 bridges (40 to 100 feet high) were involved. Average patient age was 34.8 years, $87 \%$ were male, and $29 \%$ jumped during July. These jumps/falls resulted in $25(33 \%)$ deaths and $22(29 \%)$ uninjured persons. Of those who died, $18(72 \%)$ drowned, sus taining no other detectable injuries. Hospitalized survivors and injured coroner cases suffered predominantly extremity and rib fractures, pneumo/hemothoraces, and closed head injuries. No neck injuries were identified. No patient whose advanced level EMS treatment exceeded basic monitoring and IV access survived.

Conclusions: Jumping/falling from medium height bridges into water is a survivable event. Most fatalities are attributed to drowning and not directly to other injuries. The results indicate that patients who receive extensive EMS interventions are unlikely to survive. This information may be useful for planning prevention strategies and EMS resource allocation.
60.

\section{The Impact of AEDs on Cardiac Survival in an Urban Out-of-Hospital Setting}

Harinder S. Dhindsa, $M D, M P H,{ }^{*}$ Dennis Fitzgerald, $M D$,

David Milzman, $M D$, Robert R. Bass, $M D$

Georgetown University Hospital and District of Columbia EMS, Washington, DC USA

Introduction: Prior studies have documented less than 3\% survival for out-of-hospital cardiac arrest (CA) in D.C. EMS intubation has failed to improve extremely poor survival rates for out of hospital CA. This study will investigate whether the addition of automatic external defibrillator (AED) use will improve the negligible survival rate experienced in a system that transports $80,000 \mathrm{pts} / \mathrm{yr}$; Thus replicating success with AED for CA in other centers.

Methods: Retrospective review of all CA data from D.C. EMS system from 1-12/91 (no AED) vs. 1-12/93 (AED in use). Supporting data from run sheets and hospital records was compared for years with and without the AED using student's t test, chi-square with $p<0.05$.

Results: In 1991 there were $\mathbf{4 1 4}$ out of hospital CAs arrests with an overall survival rate of $2.3 \%$ with a mean EMS arrival time of 10 minutes. There were no significant differences with respect to CA patients' age, bystander CPR or BLS/ALS response times between two years (1991 and 1993) $p=$ NS. In VF patients who comprised $26 \%$ and $24 \%$ of presenting rhythms for ' 91 and '93 respectively, the use of AEDs improved survival $3.8 \%$ vs. $29 \%$ with AEDs in ' $93(p<0.05)$. There was no difference in percentage of non-VF presenting rhythms or patient outcomes between the two groups.

Conclusion: The addition of AEDs significantly improved survival to hospital admission for out-of-hospital cardiac arrest in D.C. A multifactorial approach to faster responses, including widespread availability of AEDs may further increase survival rates. 